\title{
Design Criteria for Web Applications Adapted to Emotions
}

\author{
Giulio Mori, Fabio Paternò, and Ferdinando Furci \\ ISTI - CNR, Via Moruzzi, 1, 57126 Pisa, Italy \\ \{giulio.mori, fabio.paterno, ferdinando.furci\}@isti.cnr.it
}

\begin{abstract}
The main goal of this work is to identify a set of design criteria for Web applications taking into account the users' emotions. The results are based on the analysis of a user study with 50 participants who tested six Web interfaces, each one designed to elicit a specific emotion (hate, anxiety, boredom, fun, serenity, love). The design criteria applied to the six emotion-based Web interfaces were drawn from the results of a previous survey, which involved 57 different users, on the relationships between emotional state and Web interfaces. This initial survey asked the users to indicate the emotions most often associated with Web interaction, and then assign each emotion with some specific Web design characteristics. The resulting design criteria can form the basis for a set of emotion-related guidelines for Web application user interfaces.
\end{abstract}

Keywords: Web guidelines, emotions, affective interfaces.

\section{Introduction}

The important role of emotions in HCI is widely accepted [3-5]. However, little work has been dedicated to how to take them into account in Web applications, which are the most widely used applications. Thus, Web developers and designers need support on some design criteria (such as choice of user interface elements, navigation style, suitable colors, etc.) associated to emotional states. Emotions are complex and depend on individual preferences, attitudes, moods, affect dispositions, and interpersonal stances; "there is no single standard gold-method for their measurement" [10].

The literature reports different methodological approaches to classifying emotions, such as Geneva [8] or Feeltrace [9], and scales and questionnaires to measure either two primary (negative-positive) dimensions of moods [1], or hedonic and pragmatic dimensions of user-experience [7][12], but no work has focused on typical emotions during Web interaction. There are some works comparing different versions of Web pages [5] to investigate the impact of their attractiveness [2] or aesthetics, or if one Web site is better than another at eliciting emotions [4]. An analysis of existing Web sites about the hedonic elements (such as color, images, shapes and photographs) has been carried out [11] to investigate the emotional appeal, the sense of the aesthetic or positive impression resulting from the overall graphical look of a Website. However, none of these studies provide precise indications on how the various aspects of Web interfaces can elicit a specific emotion. Modeling the key subjective aspects of user 
experience and how it affects the perception of the final product or emotional responses [4] are important contributions, but there is the need to better address the emotion-based Web design aspects.

In this paper we aim to investigate the impact of some Web design criteria to elicit a particular emotional state on the user, independently of the application domain. In order to better understand what design choices are most suitable for most recurrent user emotions in Web applications, we have conducted two user studies described herein. Since there were no specific indications in the literature about the effects of Web design on eliciting a specific emotion, we organized a first survey to start our research, with the goal of collecting some basic indications from a sample of 57 users. The survey aimed to better understand the most recurrent emotions during Web interaction and the related Web design features. Next, we wanted to check the effectiveness of the data gathered in the survey through a user study. We applied the criteria to six Web interfaces, each one designed to elicit a specific emotion. Fifty different users judged their emotional impact. Positive emotions are certainly important for improving the user experience, however, also understanding the Web design criteria eliciting negative emotions has its importance for Web designers in order to improve their awareness of the risks and kinds of emotions their Web sites may elicit. In particular, section 2 describes the initial survey having the goal to investigate the relationships between emotional state and Web interfaces. Section 3 reports on the user study having the goal to validate the design principles through six Web Interfaces, and finally, we draw some conclusions and provide indications for future work.

\section{A Survey: Collecting Opinions about Emotional Web Design}

The questionnaire was completed by 57 users in two sessions (the average completion time per user was about two hours): the first session was carried out in the presence of the authors (to provide the users with explanations, when necessary), and a second session in which the users completed the questionnaire alone. The questionnaire was composed of three parts: 1) personal information, 2) classification of emotions in Web interaction, 3) emotion-based Web design opinions. The first part aimed to collect some personal information from the users and their experiences with the Web. In the second part, users had to propose some emotions they considered relevant during Web interaction and, for each of them, they had to freely associate colours and some attributes characterizing the user activity. The third part was more oriented to the Web design, where users had to give their opinions by associating each emotion with various Web interface features (depending on the question, we showed them various graphical examples of typical elements of a Web interface).

\subsection{Personal Information}

The participants were 25 females and 32 males, with an average age of 38,21 years (ranging from 26 to 59). Seventeen users had a $\mathrm{PhD}, 21$ users had a five-year degree, 2 users had a four-year degree, 8 users had a bachelor's degree, while 7 users had a high school diploma and 2 users had a school diploma. Users were used to surf the Internet (46 users were connected to the Web every day, 9 users navigated three times 
per week, 2 users used the Web one time every fifteen days). The sample considered both experienced and inexperienced users in Web development (at different levels, 38 users had implemented some Web interfaces, while 19 users had little or no knowledge on Web programming).

\subsection{Classification of Emotions in Web Interaction}

In this part, each user had to indicate a certain number of emotions (maximum 8) which s/he considered relevant during the Web interaction. The only constraint of their suggestions was that for each chosen emotion, they also had to indicate the opposite emotion (depending on the emotional valence, negative or positive, in their perception). The reason for this request was that we wanted to define a complete design space that given a negative emotion could allow the identification of the positive counterpart. At the beginning of the questionnaire, some users found difficulties to define emotions and needed support, however at the end, the total average number of the proposed emotions was 3.84 per user, with a total of 219 distinct proposed emotion names perceived as negative and other 219 perceived as positive emotions. Each user had to associate a value (in an ordered scale from -8 to +8 ) to each emotion, as a measure to indicate how negative or positive s/he considered it. Analyzing the proposed emotions of the 57 users, we decided to consider as more "significant" only the emotions with at least 10 preferences, discarding the remaining others, which had received just only 1, 2 or at maximum 3 preferences each one. In addition, after having analyzed the complete final results of the questionnaire, we noticed that some emotions (having different proposed names by the users, but similar meaning), were characterized by the same Web features. In these cases, with the consent of the users who proposed the emotions, synonyms have been considered the same emotion, and finally, we have obtained an essential basic ordered scale of 6 emotions ( 3 negative and 3 positive) to express the typical affective states of a user interacting with Web (see Table 1), corresponding to well distinguishable Web design characteristics. The final emotions would have been the same also first joining the synonyms and then filtering the emotions with at least 10 preferences because in addition to the "primary emotions" [13] (such as hate, love, etc.) many users proposed a lot of different "secondary emotions" [13] (such as jealousy, nostalgia, loneliness, etc.), which were proposed only by few other users.

Considering the complexity of the emotions world for human beings and the many emotion classifications existing in literature [8][9][10] (even if no one is specifically oriented to the Web interaction and design), this classification is not exhaustive. However, the goal of this work is not to provide a further emotion classification, but rather to investigate whether some clearly distinguishable design characteristics can elicit a specific emotion on the user independently of the application domain. So this small starting set composed of 6 emotions oriented to Web interaction (obtained by the users suggestions) has been the basis of our study. The goal is to understand if some different Web design features can concretely have a specific impact on the users perception and on the personal emotional state, independently of the contents. Looking at Table 1, some considerations about the meanings of the emotions in the scale proposed by the users are necessary: a) hate and love express the sense of disliking/liking or indifference/empathy for something or somebody (typical of Web social 
network environments and Web 2.0). English language expresses well these meanings (i.e.: I hate/love Louis Armstrong music); b) anxiety and serenity express the emotional state during critical/safe actions (i.e.: the user is booking/buying something on Web and s/he need to fill a form inserting personal or credit card data); c) boredom or fun depends on the interest of users for the Web content, and the way the contents are presented is fundamental. Table 1 shows the ordered scale of the emotions with the corresponding average values proposed by the users.

Table 1. The main 6 emotions indicated by the users. The order is determined by the average values assigned by the users on the scale of a negative or positive personal perceptions.

\begin{tabular}{|c|c|c|c|c|c|}
\hline \multicolumn{5}{|c|}{ Average values of the main Web Emotions considered relevant by the users } \\
\hline Hate & Anxiety & Boredom & Fun & Serenity & Love \\
\hline-3.48 & -2.54 & -1.99 & +2.1 & +2.25 & +3.6 \\
\hline Standard Deviation values of the main Web Emotions considered relevant by the users \\
\hline 1.29 & 1.04 & 0.76 & 0.92 & 0.83 & 1.23 \\
\hline
\end{tabular}

In this part of the questionnaire, we also asked the subjects to associate some attributes related to the user activities for each emotion. Users had to select from the following pairs: static or dynamic (perceived level of changes of the interfaces reflecting the changes of the personal emotional state), passive or active (perceived level of the user's involvement in doing actions), simple or complex (perceived level of how the interface can be elaborated in design). The results are summarized in Table 2. As a criteria of choice (for the results of Table 2 and for the other tables presented for this first survey), we took into considerations an attribute as strongly characterizing an emotion, when the total number of users' preferences for a value of a pair (or group) was at least the double (over 50\%) of the other/s preferred choices; in borderline cases, conclusions could be ambiguous. The double threshold was a prudential strategy, because we noticed that when a characteristic was chosen quite unanimously by most of the users, that characteristic collected a number of preferences higher than the double (in comparison with the other choices).

Table 2. User activity attributes for each emotion

\begin{tabular}{|c|c|}
\hline Emotions & User activities attributes \\
\hline Love & dynamic, active, complex \\
\hline Serenity & static, simple \\
\hline Fun & dynamic, active \\
\hline Boredom & static, passive, simple \\
\hline Anxiety & dynamic, complex \\
\hline Hate & static, active \\
\hline
\end{tabular}

The following part of the questionnaire asked also to the users to associate one or more colors (preferably belonging to the 16 HTML standard color palette supported by all browsers [6]) to each emotion, and then it was asked to associate freely some visual characteristics, real objects or abstract ideas. The exact tint of the 16 colors were showed to each user during the questionnaire. Table 3 summarizes the results. 
Table 3. Colours and visual characteristics for each emotion

\begin{tabular}{|c|c|c|}
\hline Emotions & Main Colors & Visual Characteristics \\
\hline Love & red, pink & bright, transparent, indefinite, heart \\
\hline Serenity & blue, aqua, white, green, lime & $\begin{array}{c}\text { clear, bright, calm waters, large open spaces, } \\
\text { open sky, nature, flat, smooth, light colors }\end{array}$ \\
\hline Fun & $\begin{array}{c}\text { fuchsia, red, orange, yellow, } \\
\text { green, lime, teal, aqua }\end{array}$ & $\begin{array}{c}\text { brilliant, bright, colorful, spring, light, sun, } \\
\text { flowers, light fire }\end{array}$ \\
\hline Boredom & silver, gray, black & $\begin{array}{c}\text { night, dark, blurred, indefinite, hazy, fog, } \\
\text { opaque, rain, tears, dim, empty }\end{array}$ \\
\hline Anxiety & black, gray, navy, yellow & $\begin{array}{c}\text { night, dark, wavy, intermittent, storm, throb- } \\
\text { bing, blurred, indefinite, fog }\end{array}$ \\
\hline Hate & black & night, dark \\
\hline
\end{tabular}

\subsection{Emotion-Based Web Design}

The last part of the questionnaire asked the users suggestions regarding specific associations between each emotion and many Web design features. This part aimed to investigate the structure, the multimedia elements and type of interactive elements being the most effective to evoke a specific emotional state. The users could express their preference about any elements of the interface or Web design features, (we showed for each question some graphical samples with the goal that each proposed choice was clear for the users). In particular, first the users had to choose between the following groups related to the Web site structure and the interaction elements for data insertion: 1) few or many pages, 2) blurred or clear text, 3) short or long text, 4) presence of textbox (to insert short data) or textarea (to insert long information, e.g. requests). Table 4 summarizes the results.

Table 4. Page contents \& structure for each emotion

\begin{tabular}{|c|c|}
\hline Emotions & Pages \& Content structure \\
\hline Love & few pages, long text, clear text, textarea \\
\hline Serenity & little content, short text, clear text, textarea \\
\hline Fun & many pages, short text, clear text, textbox \\
\hline Boredom & few pages, long text, textbox \\
\hline Anxiety & many pages, blurred text, textarea \\
\hline Hate & few pages, blurred text, textbox \\
\hline
\end{tabular}

We then asked the users their opinions about the emotional impact of the multimedia element style, whereby the users had to choose one option from each group: 1) presence of video, animations, images, or no multimedia, 2) small, medium or large size of the images, 3) definition of the images (blurred or clear), 4) color, black \& white, or de-saturated (the color was reduced) images. Table 5 shows the results. It is not trivial at all (we cannot say if it depends on some cultural factors or something unconscious in human beings), observing that every user suggested unanimously color and clear images for every positive emotion, and blurred and no color images for two negative emotions hate and anxiety (for boredom absence of images or video was perceived by the users as a factor more emphasizing boredom). 
Table 5. Multimedia elements style for each emotion

\begin{tabular}{|c|c|}
\hline Emotions & Multimedia Elements \\
\hline Love & color medium/large clear images \\
\hline Serenity & color medium-clear images, videos \\
\hline Fun & animations, color medium-clear images, videos \\
\hline Boredom & no images, no videos \\
\hline Anxiety & de-saturated small blurred images, videos \\
\hline Hate & black \& white medium/large blurred images, videos \\
\hline
\end{tabular}

The questionnaire also sought to explore the users' opinions about the navigation elements (Table 6), choosing amongst the following options: 1) links, standard buttons, graphic buttons or tabs, 2) static or dynamic effects on navigation elements.

Table 6. Navigation elements for each emotion

\begin{tabular}{|c|c|}
\hline Emotions & Navigation Elements \\
\hline Love & graphic buttons, dynamic effects \\
\hline Serenity & tabs, link, graphic/standard buttons \\
\hline Fun & graphic buttons, dynamic effects \\
\hline Boredom & links/standard buttons, static effects \\
\hline Anxiety & standard/graphic buttons, dynamic effects \\
\hline Hate & standard/graphic buttons, static effects \\
\hline
\end{tabular}

Finally, the users gave their opinions about the interactive elements (Table 7), choosing from among the following options: 1) interactive elements with static or dynamic effects 2) textual or graphic interactive elements, 3) radio-button or pulldown single selection, 4) checkboxes, scroll or fixed multiple selection.

Table 7. Interactive elements for each emotion

\begin{tabular}{|c|c|}
\hline Emotions & Interactive Elements \\
\hline Love & dynamic, graphic, pull-down, checkboxes/scroll selection \\
\hline Serenity & static, textual \\
\hline Fun & dynamic, graphic \\
\hline Boredom & static, textual, checkboxes \\
\hline Anxiety & dynamic, graphic, pull-down menu, scroll select \\
\hline Hate & checkboxes \\
\hline
\end{tabular}

As a confirmation of the complexity of the emotions, it is not surprising that tables contain some overlapping Web features between the six emotions. With this survey we received indications about many other aspects of Web design (such as position of the elements, font type/dimension, alignment of text, etc.). We do not report these extra data, because the results are too ambiguous and further tests are necessary. 


\subsection{Additional Emotion-Related Design Aspects}

In addition to the analysed data, we received interesting comments of the participants suggesting us useful indications to improve the emotional effect of Web design. Even if enumeration of these comments was not possible because some users did not provide any comment, we decided to apply them to the Web interfaces design (see section 3) to verify their effects. We report below the summary of the key indications:

Hate-Love. A user can feel hate due to the design of the interface, if the interface is difficult to use (bad usability), the layout of the interface and/or the positioning of the widgets are confused (not easy to understand). In the worst case, the interface obstacles the interaction of the user, or/and something is not working (i.e. elements of the interface are not responding to the user input). On the contrary, a user can feel love due to the design of the interface, if the appealing aesthetic of the Web interface stimulates its use. Besides, the interface should be easy to use (good usability), the layout and disposition of the widgets should be disposed in a way easy to understand.

Anxiety-Serenity. A user can feel anxiety due to the design of the interface, if the interface emphasizes particular stress factors (i.e. a deadline, risk, or sense of losing something, etc.). In these stress conditions, the interface does not allow the user to reason comfortably (i.e. adding intermittent light effects, distortion or jerky transformations of the elements in the interface, etc.). On the contrary, a user can feel serenity due to the design of the interface, if the interface let the user to feel safe, (i.e. providing always feedbacks, or showing well known reassuring elements, as a logo of secure transactions, etc.). Besides, the simplicity of the interface allows the user to interact easily, reducing her/his effort and giving the time s/he needs.

Boredom-Fun. A user can feel boredom due to the design of the interface, if the interface provides or requires lots of information (i.e. very long texts, or many required fields in a form, etc.). Much text without images or multimedia elements, increases boredom. On the contrary, a user can feel fun due to the design of the interface, if unexpected elements, animations or effects surprise the user in a positive way. The animations or dynamic effects should be oriented to facilitate the interaction, otherwise they are perceived as annoying.

\section{A User Study: Applying the Emotion-Based Design Principles}

On the basis of the results of the survey, we developed six Web interfaces, applying the collected Web designs principles. Each Web interface had the goal to elicit one of the six emotions of the scale. Each Web interface presented the same content (except very minimal additions suggested by the users) in a different design style. Considering that most users in the survey suggested music as topic for an emotion-based Web 
application, we chose the Beatles' musical history as a topic for the Web six interfaces. In details, the interfaces contained: a short textual biography, a media player to listen to five famous songs, a musical video, a form where the user could buy some virtual tickets for revival musical events, and some clickable graphical covers of six famous albums. Finally, we recruited 50 different users (through a mailing list of our institute), who had not participated to the previous survey, to test the six Web interfaces through some interactive tasks, and after that, to judge (through a questionnaire) the Web design effectiveness in stimulating a specific emotion.

\subsection{Description of the User Test and Discussion of the Results}

We showed the six Web interfaces to the 50 users in random order. We wanted to avoid that the order could influence the emotional perception. Each user had to test each Web interface by completing three tasks, and then s/he had to fill in an online questionnaire. The questionnaire was composed of three sections, and it asked the users: a) personal information, b) judgment about the effectiveness of the interface to stimulate the proposed emotion and comments, c) suggestions about other emotions we did not consider in the classification, opinions about the utility of the adaptation of the Web design to elicit more positive emotions on the users, and some suggestions about useful application fields. Most users considered the six emotions exhaustive for the Web interaction (even if some users proposed anger or surprise as examples of additional emotions). Nearly all the participants found the adaptation of the Web design to elicit positive emotions very useful. As important applications for applying the emotional Web design, they suggested educational environments, telemedicine and online psychological platforms, games, home automation applications, or tools oriented to helping people with disabilities or the elderly. The users preferred Web design stimulating positive emotions because it improves the user experience. However, they also considered the utility of eliciting negative emotions not only to improve the awareness of Web designers, or to recreate particular thrilling atmospheres in games, but also in the telemedicine field. In this area the ability to understand the reactions of patients in a good or bad affective state is important. Moreover, it could improve children's awareness about the difference between good and bad behavior in some educational or learning tools. The users gave their judgment in a scale from 1 to 5 (where the value 1 indicated that the page was very ineffective to elicit the proposed emotion, while value 5 indicated that the page was very effective, and the value 3 represented the neutrality). Considering that the survey was "open answer" (concerning the users' proposal of Web emotions), we decided for this user test that the users could know in advance the emotional goal of the currently tested interface. We wanted to avoid that another "open-answer user test" could produce too vague results. In particular for each emotion, the users were asked to accomplish three tasks: a) find the answer to a proposed question in the biography text (the goal was obliging the user to read the text with specific style characteristics), b) click on one proposed cover of one album (the goal was obliging the user to evaluate the interaction with the elements of navigation), c) fill in a form (with proposed data corresponding to one imaginary user) to buy some virtual tickets (the goal was obliging the user to evaluate 
the elements of interaction). The questionnaire was completed by 50 users (average completion time per user of about one hour). The participants were 21 females and 29 males, with an average age of 38,28 years (ranging from 26 to 77). Ten users had a $\mathrm{PhD}, 21$ users had a five-year degree, 4 users had a four-year degree, 9 users had a bachelor's degree, while 6 users had an high school diploma. Users were used to surf the Internet (42 users were connected to the Web every day, 5 users navigated three times per week, 2 users used the Web one time every fifteen days, and one used Web when it happens). The sample considered both experienced and inexperienced users in Web development (at different levels, 28 users had implemented some Web interfaces, while 22 users had little or no knowledge on Web programming).

The six Web interfaces were designed as follows: a) the Web interface to elicit hate was completely unusable with a confused layout, b) the Web interface to elicit anxiety showed intermittent light effects and jerky transformations, with a countdown as a pressure factor to fill in the form, c) the Web interface to elicit boredom, was neutral, without images or videos, requiring more fields to fill in the form, d) the Web interface to elicit fun, showed unpredictable animations and dynamic effects, e) the Web interface to elicit serenity, was very simple to minimize the user's effort, f) the Web interface to elicit love had an appealing graphics and it was usable. For lack of space, we have to omit further details. The average judgment of the 50 users about the effectiveness of the interfaces to elicit the proposed emotion was high: (over value 4) for hate, anxiety, boredom and serenity, while it was slightly effective (over value 3 ) for fun and love. The results of the user test showed that the usability, even if it is an important factor, it is not the unique aspect responsible to elicit an emotional reaction on the user (e.g. the different unusable interfaces aiming to elicit hate and anxiety, produced different emotional effects). Even if the results are encouraging, a more detailed statistical analysis is necessary. In particular it is important to understand for each emotion, if a subset ("core") of key design factors can be responsible for eliciting a specific mood. It is necessary investigate further if the results can depend on some "confounding factors" (such as age, gender, experience in development, etc.), or if the results are domain/topic-dependent or domain/topic-independent.

\section{Conclusions and Future Work}

The goal of this work has been to study whether the collected preliminary user indications about the relevant emotions during Web interaction and the corresponding specific design criteria actually do have an emotional impact on users. The results obtained are encouraging, even if further refinements and investigation are necessary. In particular, other user tests will be necessary to better understand the essential Web design key factors affecting emotional state. The ultimate goal of this research is the formalization of a set of Web guidelines to design interfaces that can effectively stimulate user emotions during the interaction. For the implementation of the next user tests, we are considering various sensors to detect physiological parameters of the users in order to monitor their changes and to adapt the Web design with the goal of eliciting more positive emotions on the users. 


\section{References}

1. Watson, D., Clark, L.A.: Development and Validation of Brief Measures of Positive and Negative Affect: the PANAS Scales. Journal of Personality and Social Psychology 54(6), 1063-1070 (1988)

2. Hartmann, J., Sutcliffe, A., De Angeli, A.: Investigating Attractiveness in Web User Interfaces. In: Proc. CHI 2007. ACM Press (2007)

3. Hassenzahl, M.: The Think and I: Understanding the relationship between user and product. Funology Human Computer Interaction Series 3, 31-42 (2005)

4. Karlsson, M.: Expressions, Emotions, and website design. CoDesign 3(1), 75-89 (2007)

5. Kim, J., Lee, J., Choi, D.: Designing Emotionally Evocative Homepages: An Empirical Study of the Quantitative Relations Between Design Factors and Emotional Dimensions. International Journal of Human-Computer Studies 56(6), 899-940 (2003)

6. The 16 HTML color names, http: / / en.wikipedia .org/wiki/Web_colors

7. Voss, K.E., Spangenberg, E.R., Grohmann, B.: Measuring the hedonic and utilitarian dimensions of consumer attitude. Journal of Marketing Research 40(3), 310-320 (2003)

8. Bànziger, T., Tran, V., Scherer, K.R.: The Geneva emotion wheel. Journal, Social Science Information 44(4), 23-34 (2005)

9. Cowie, R., Douglas-Cowie, E., Savvidou, S., McMahon, E.: Feeltrace: an instrument for recording perceived emotion in real time. In: Proceedings of the ISCA Workshop on Speech and Emotion 2000, pp. 19-24 (2000)

10. Scherer, K.R.: What are emotions? And how can they be measured? Social Science Information 44(4), 695-729 (2005)

11. Cyr, D.: Emotion and Website Design, 2nd edn., ch. 40., http: / / www . interaction-design.org/encyclopedia/ emotion_and_website_design.html

12. Diefenbach, S., Hassenzahl, M.: The Dilemma of Hedonic - Appreciated, but hard to justify. Interacting with Computers 23(5), 461-472 (2011)

13. Damasio, A.R.: Descartes' error: Emotion, reason, and the human brain. Avon Books, New York (1997) 